\title{
Research on the Parameters of Tailings Cemented Filling for Mined-out Open Pits
}

\author{
Liang Peng ${ }^{1,}$, Luhong Jian ${ }^{1, b}$ \\ ${ }^{1}$ College of Mining Engineering in North China University of Science and Technology, Tangshan, \\ 063009, China \\ aemail: andyliang1990@163.com, bemail: luhongjian@ncst.edu.cn
}

Keywords: Mined-out Open Pit; Filling Parameter; Orthogonal Experiment; Finite Element Analysis

\begin{abstract}
Taking the treatment project of tailings cemented filling for mined-out open pits in typical metal mines as background, using theoretical analysis and numerical simulation research method, the cement-sand ratio and concentration of filling slurry, the total filling height, level height, stratification height and the methods to determine the structure parameter of cement-sand ratio at different stages were provided. And then through the calculation and analysis, it is concluded that the cement-sand ratios of filling slurry of mined-out open pits are classified into three different grades: $1: 8,1: 10,1: 20$; the slurry concentration is $70 \%$; the filling height is $80 \mathrm{~m}$; the level height is $20 \mathrm{~m}$; the stratification height is $1.5 \mathrm{~m}$; the cement-sand ratios of filling slurry of four stages are 1:8, 1:8, 1:10, 1:20.
\end{abstract}

\section{Introduction}

With the gradual depletion of shallow resources, many mines face a specific period of the transition from open pit to underground mining. At present the mining-out area at the bottom of open pit has great influence on the slope stability by the method of open stope mining after the transition from open pit to underground mining. At the same time the catchment of open pit penetrates into underground through the joints and fissures of surrounding rocks during the rainy season, which not only increases drainage costs, but also causes deterioration of surrounding rock, and it has a significant impact on mine safety. Whether can solve the open mining pit legacy security problems reasonably has become the guarantee for mining safety production [1-4].

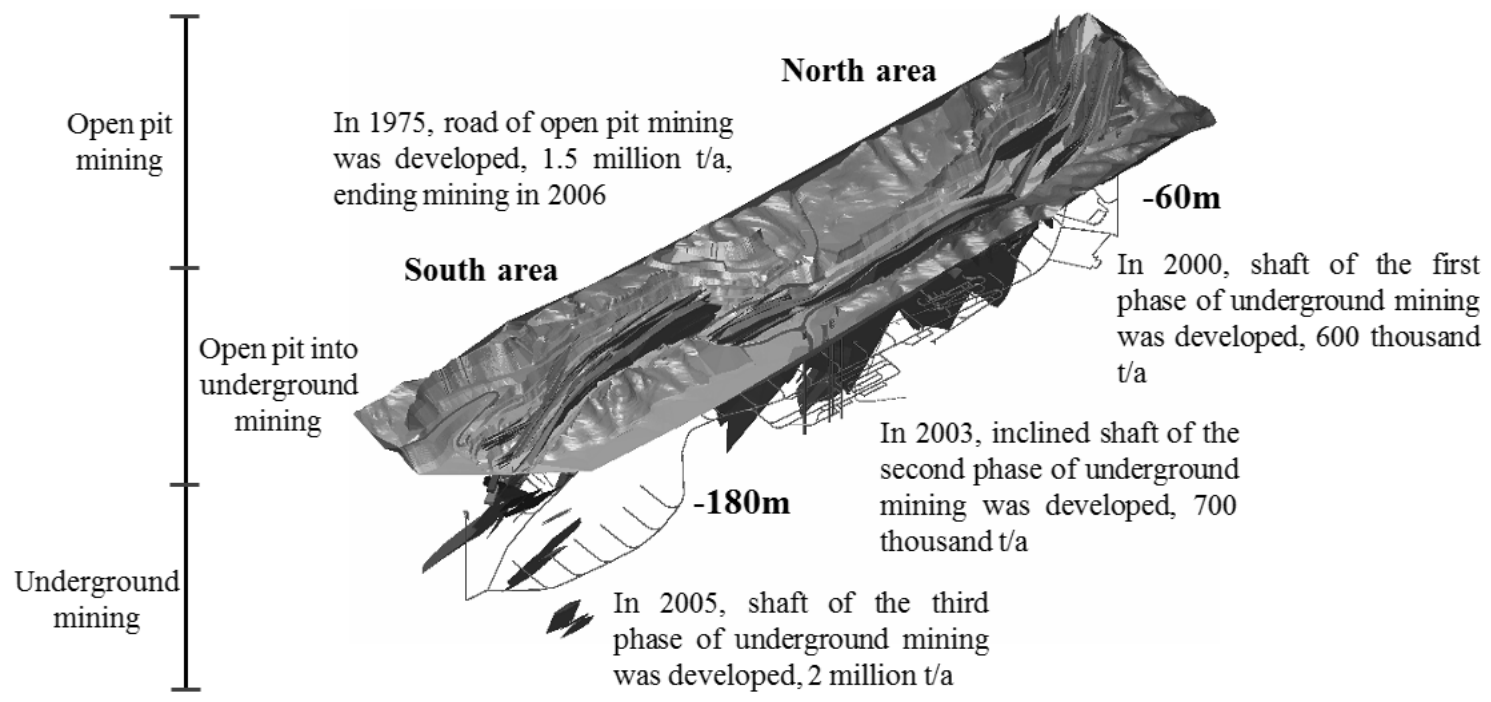

Fig.1. The spatial distribution

In the early years, open-pit mining is adopted in Shirengou Iron Mine, the bottom of open pit is ended in 0m level. The first phase construction from open pit to underground was began in 2000. The shallow hole shrinkage mining method was used to mine the orebody of south area at the medium stage of $0 \sim-60 \mathrm{~m}$. In 2003, The second phase construction was began and the shallow hole 
shrinkage mining method was implemented to mine the orebody of north area at the medium stage of $-16 \sim-60 \mathrm{~m}$. In 2005, The third phase construction was began and the sublevel open stoping with subsequent backfilling method was implemented to mine the orebody at the medium stage of -60 $-180 \mathrm{~m}$ [5]. The spatial distribution is shown in Figure 1.

Open-stope mining method is adopted in transitional period, about $25 \mathrm{~m}$ boundary pillar was left. In order to ensure the safety of open-pit slope and the underground production, the stope pillars at -60m level were not recovered. Boundary pillars and stope pillars were about 1.8 million tons. There had been formed about 1.25 million cubic meters mined areas at $-60 \mathrm{~m}$ level, which had be filled after the establishment of the filling system.

Though filling mining method was adopted in underground mining, the mined areas would still be existed for a period of time because of delayed of filling. Moreover filling mining method can ensure no large-scale collapse of the ground but not to guarantee sedimentation of the surface. So deep-underground mining still has a great influence on the mining-out area.

The 20 and 180 meters high slope had experienced multiple disturbance during underground mining, marginal ore mining, open-pit mining, which cause serious deterioration of surrounding rock mechanical property, and local slope might be about to destroy. Deep-underground mining may trigger collapse of slope, which affects the stability of surface development system, filling station, underground roadways and stopes.

Although open stoping with subsequent backfilling consumed large amounts of tailings, there were 80000 cubic meters tailings cannot be used and the tailings pond would be closed.

Based on the analysis, using theoretical analysis and numerical simulation research method, the cement-sand ratio and concentration of filling slurry, the total filling height, level height, stratification height and the methods to determine the structure parameter of cement-sand ratio at different stages were provided.

\section{The determination of cement-sand ratio and concentration}

Tailing-cemented filling slurry of open pit is from filling station. When the cement-sand ratio provided by mine filling system is less than about $1: 8$, filling slurry concentration is about $70 \%$, the system can work steadily. The selection of cement-sand ratio is three grades, which is $1: 20,1: 10$ and $1: 8$, and slurry concentration is $70 \%$.

\section{The determination of total filling height}

The storage of excess tailings and the elimination of potential danger of open pit slope should be considered comprehensively when the filling height of open pit mining-out area is about to determined. Typical profile of open pit slope danger zone is chosen, finite element theory is used to analyze the stability and delineate top level of potential slip region, as a result, the treatment height $H_{1}$ (in the case of horizontal displacement, as shown in Figure 2) is 60 meters.

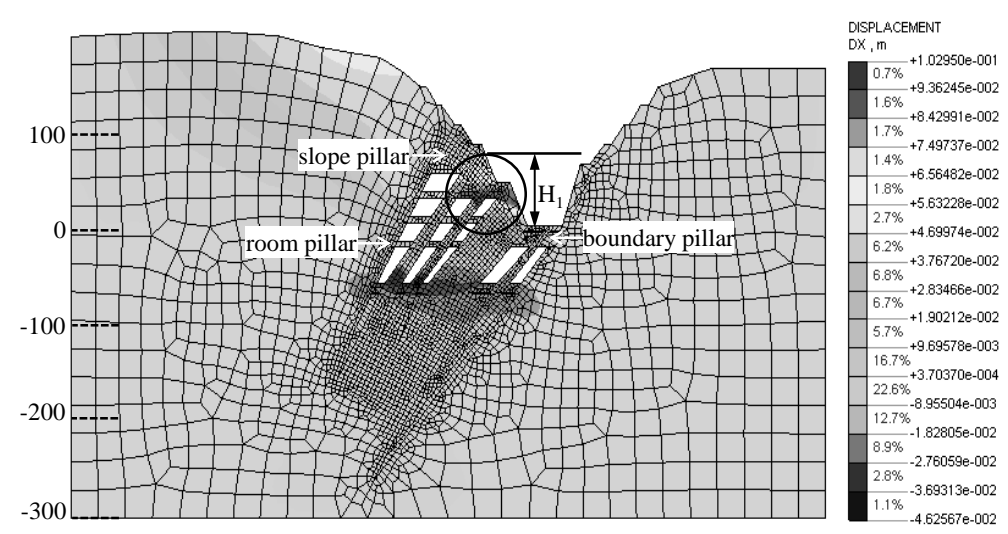

Fig.2. Horizontal displacement analysis chart of typical profile of mine

The amount of surplus tailings can be calculate according to mining method, production capacity 
and service year, the height of $H_{2}$ is $80 \mathrm{~m}$ based on the present situation of open pit. The total filling height is 80, which is the maximum value between $H_{1}$ and $H_{2}$.

\section{The determination of level height}

The determination of level height of treatment of open pit by cementing filling is on the basis of considering recycling boundary pillar resources, which is calculated by using the "three zone" theory. In order to guarantee the output connection, about 22 meters thick boundary pillar is set up during the transition from open-pit to underground mining.

Recycling height is about $3.4 \mathrm{~m}$ when upward horizontal cut-and-fill stoping method is adopted. The cavity height which also be called filling height of not pick up the top is $0.5 \mathrm{~m}$.

(1) The height of caving zone is calculated [6], $H_{\text {caving }}, H_{\text {caving }}=H_{\text {cavity }} /(K-1)$. The coefficient of volume is $1.5, H_{\text {caving }}$ is $1.0 \mathrm{~m}$.

(2) The height of fissure zone is calculated according to cavity height [7], $H_{\text {fissure, }}$, $H_{\mathrm{d}}=A H_{\text {cavity }} /\left(B H_{\text {cavity }}+C\right)$, A takes 100 , B takes 0.85 , C takes $6.2, H_{\text {fissure }}$ is $7.55 \mathrm{~m}$.

(3) The level height, $H_{\text {stage }}, H_{\text {stage }}=D\left(H_{\text {caving }}+H_{\text {fissure }}\right)$, D is a safety factor, which is $2.3, H_{\text {stage }}$ is $19.6 \mathrm{~m}$, but $20 \mathrm{~m}$ is selected.

(4) The number of stages is determined as four according to the total filling height, $H_{\text {stage } 1 \text { 、 }}$ $H_{\text {stage2 }} 、 H_{\text {stage3 }} 、 H_{\text {stage } 4}$, as shown in Figure 3.

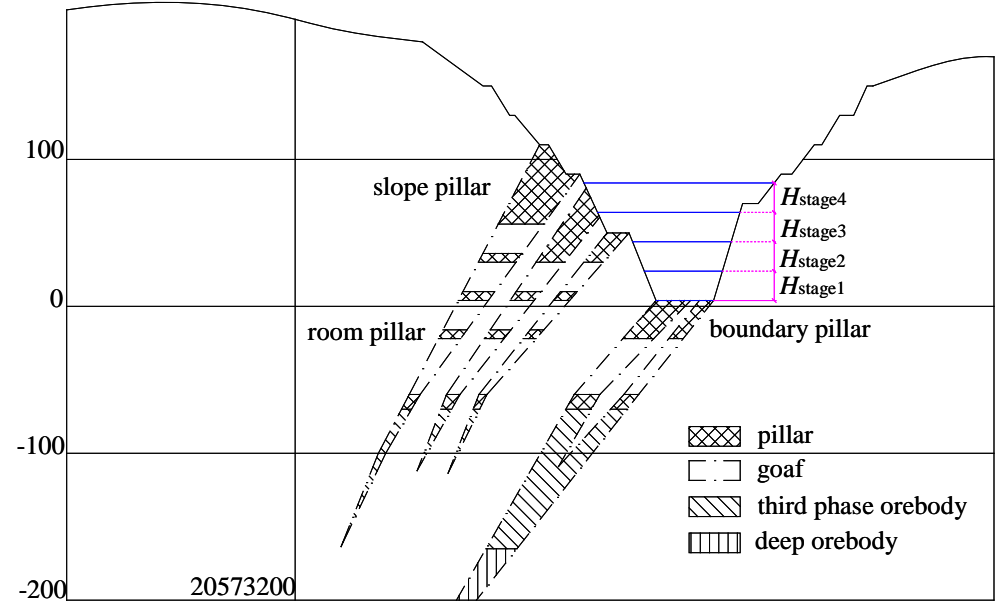

Fig.3. Stage distribution chart of cemented filling of open pit

\section{The determination of stratification height}

According to the ability of tailings emission and consumption, the surplus amount of tailings is calculated, at the same time based on the construction of the water treatment patio (a construction height is $1.6 \mathrm{~m}$ ) the filling stratification height is determined as $1.5 \mathrm{~m}$. The construction sequence is to build a $1.6 \mathrm{~m}$ water treatment patio firstly, secondly fill the cement slurry of $1.5 \mathrm{~m}$, last fill the cement slurry of $1.6 \mathrm{~m}$ after the filling body meets the design strength, this steps cycle.

\section{The determination of filling slurry cement-sand ratio in different stages}

According to the number of stages and cement-sand ratio level, the different schemes of cement-sand ratio are determined by using orthogonal experiment theory. Finite element method is used to calculate the different filling schemes and the stability of the underground ore-body mining, the open pit boundary pillar after recovery, the plastic zone index is extracted as evaluation index to determine the reasonable solution.

The number of filling stages of open pit is four, $H_{\text {stage } 1}$ as the influence area of boundary pillar mining, the cement-sand ratio is $1: 8$, the optional class of cement-sand ratio of $H_{\text {stage } 2} 、 H_{\text {stage } 3} 、 H_{\text {stage } 4}$ is $1: 8 、 1: 10 、 1: 20$. According to the principle of orthogonal test [8], the three different filling stages 
are regarded as three factors, three different cement-sand ratio are considered as three levels. Calculation scheme is determined by orthogonal table which includes the three factors and three levels. There are nine set of filling schemes, shown in table $1 \sim 2$.

Table 1 Value of cement-sand ratio of different filling intervals

\begin{tabular}{cccc}
\hline Levels & $20 \sim 40 \mathrm{~m}$ & $40 \sim 60 \mathrm{~m}$ & $60 \sim 80 \mathrm{~m}$ \\
\hline 1 & $1: 8$ & $1: 8$ & $1: 8$ \\
2 & $1: 10$ & $1: 10$ & $1: 10$ \\
3 & $1: 20$ & $1: 20$ & $1: 20$ \\
\hline
\end{tabular}

Table 2 Scheme of cement-sand ratio of different filling intervals

\begin{tabular}{ccccc}
\hline Schemes & $0 \sim 20 \mathrm{~m}$ & $20 \sim 40 \mathrm{~m}$ & $40 \sim 60 \mathrm{~m}$ & $60 \sim 80 \mathrm{~m}$ \\
\hline 1 & $1: 8$ & $1: 8$ & $1: 8$ & $1: 8$ \\
2 & $1: 8$ & $1: 8$ & $1: 10$ & $1: 10$ \\
3 & $1: 8$ & $1: 8$ & $1: 20$ & $1: 20$ \\
4 & $1: 8$ & $1: 10$ & $1: 8$ & $1: 20$ \\
5 & $1: 8$ & $1: 10$ & $1: 10$ & $1: 8$ \\
6 & $1: 8$ & $1: 10$ & $1: 20$ & $1: 20$ \\
7 & $1: 8$ & $1: 20$ & $1: 8$ & $1: 8$ \\
8 & $1: 8$ & $1: 20$ & $1: 20$ & $1: 10$ \\
9 & $1: 8$ & $1: 20$ & & \\
\hline
\end{tabular}

On the basis of typical profile (Figure 2), the finite element theory is used to simulate different filling schemes, distribution situation of the plastic zone of open pit after different filling schemes is shown (shown in Figure 4, the results of scheme 9 is not convergent after calculation so it will not be analyzed). Local failure of cementing filling body is allowed (producing the plastic zone), but water channel can not be formed. At the same time filling cost should be considered, and the different solutions will be optimized based on this principle.

The black is the elastic zone and the white is plastic zone in Figure 4. Through the comparative analysis of plastic zone of different schemes, it can be seen that, when filling slurry whose cement-sand ratio is 1:8 is adopted at the first cemented filling stage of open pit, there are no plastic zones. When filling slurry whose cement-sand ratio is 1:8 is adopted at the second cemented filling stage of open pit, there are no plastic zones.( as scheme 1 3 shown in Figure 4), plastic penetration area is produced when the cement-sand ratio is $1: 10$ and 1:20( as scheme 4 8 shown in Figure 4). When filling slurry whose cement-sand ratio is 1:8 is adopted at the third backfill stage of open pit, there are no plastic zones( as scheme $1,4 、 7$ shown in Figure 4), local plastic zone is produced when the cement-sand ratio is 1:10( as scheme 2、5、8 shown in Figure 4), plastic penetration area is produced when the cement-sand ratio is 1:20( as scheme 3 and 6 shown in Figure 4). When filling slurry whose cement-sand ratio is 1:8 is adopted at the forth backfill stage of open pit, there are no plastic zones( as scheme 1、6、8 shown in Figure 4), small plastic zone is produced when the cement-sand ratio is 1:10( as scheme 2 and 4 shown in Figure 4), local plastic zone is produced when the cement-sand ratio is 1:20( as scheme 3、5、 7 shown in Figure 4).

Based on the analysis above, filling slurry cement-sand ratio of different stages of open pit is finally determined: the ratio of $H_{\text {stage } 1}$ and $H_{\text {stage2 }}$ are 1:8, $H_{\text {stage3 }}$ is $1: 10$ and $H_{\text {stage4 }}$ is $1: 20$. 

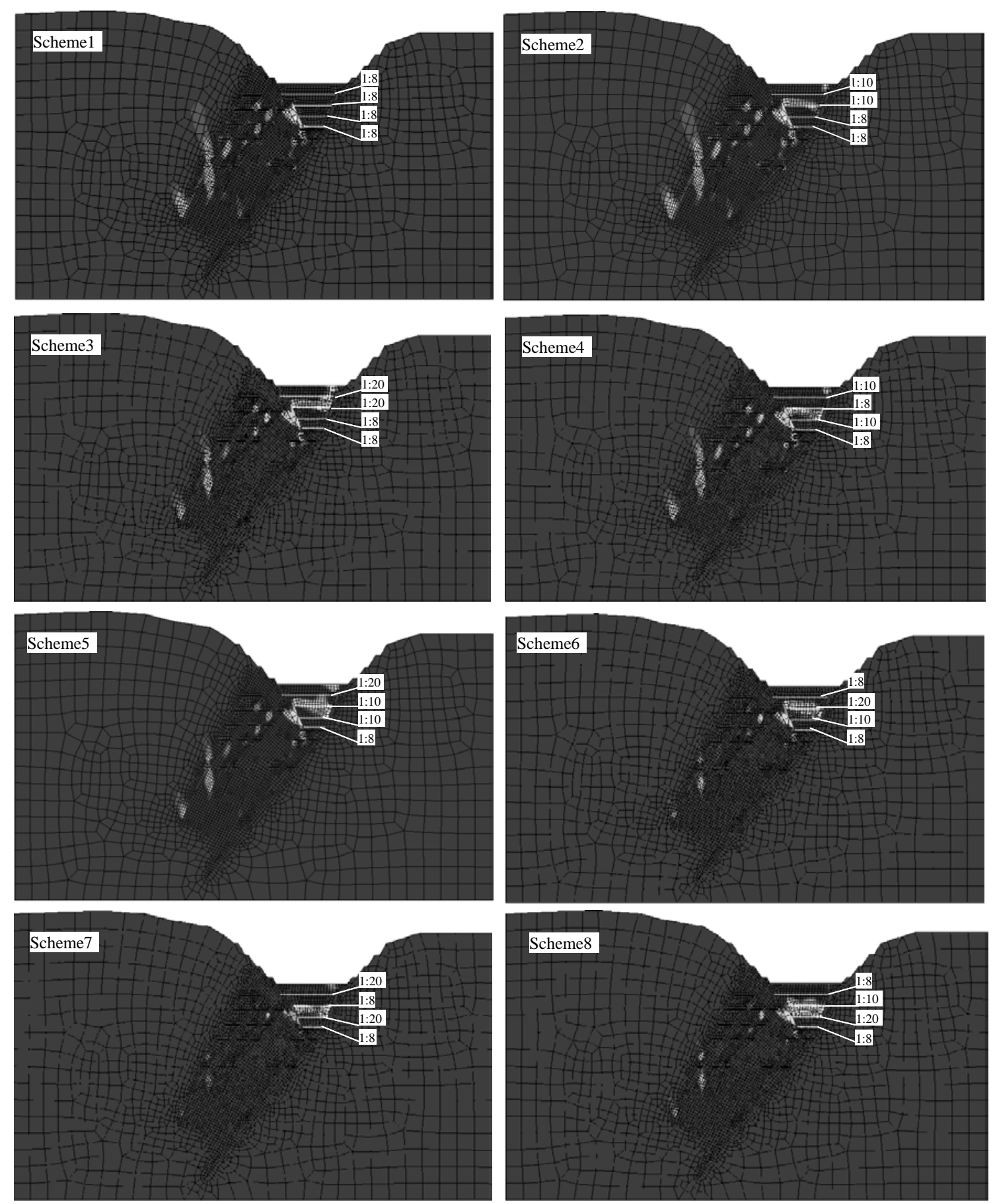

Fig.4. Plastic area distributions of different treatment schemes of cemented filling for open pits

\section{Conclusion}

(1) Considering filling slurry cement-sand ratio and concentration of synergy filling station system, the cement-sand ratios are three grades which are 1:20, 1:10 and 1:8, the slurry concentration is $70 \%$.

(2) Cementing filling height is determined to $80 \mathrm{~m}$ that bases on the slope stability analysis and the capacity calculation of open-pit mining tailings.

(3) On the basis of considering the recycling of boundary pillar, the level height of treatment of open-pits by cementing filling is identified as 20 meters by using the "three zone" theory. According to the tailing emissions and construction of the draining patio, layer height is determined to $1.5 \mathrm{~m}$.

(4) The filling material cement-sand ratio of 4 stages $(1: 8,1: 8,1: 10,1: 20)$ is determined by orthogonal experiment and the finite element theory. 


\section{Acknowledgement}

In this paper, the research was sponsored by the Nature Science Foundation of Hebei Province (Project No. E2014209093).

\section{References}

[1] Lian Jie, Zheng Maoxing, Wu Fei,et al. Research of treatment and comprehensive utilization of open pits[J]. Metal Mine, 2013(6):134-137.

[2] Wang Yubo, Wang Yunmin, Wang Bin,et al. Research of cementing filling scheme for a goaf[J]. Metal Mine, 2013(3):6-9.

[3] Zhao Haijun, Ma Fengshan, Guo Jie,et al. Effect of open-pit to underground mining on slope stability in Longshou Mine[J]. Journal of China Coal Society, 2011, 36(10):1635-1641.

[4] Dai Yongxin. Discussion on key technologies of tailings pond reconstruction from open pit[J]. Metal Mine, 2012(1):58-62.

[5] Zhang Jianyong, Su Jianjun. Mining method selection for third phase project of Shirengou Iron Mine[J]. Metal Mine, 2013(1):23-26.

[6] Lu Hongjian, Gan Deqing. Stability comprehensive analysis model of retained goafs of iron deposit[J]. Metal Mine, 2013(3):62-65.

[7] Wang Chunguang. Determination of overlying strata caving zone and fractured zone height[J]. Opencast Mining Technology, 2013(3):30-35.

[8] Fu Hongyuan, Liu Jianhua, Zhang Li,et al. Dynamic stability analysis for rock slope based on orthogonal test[J]. Journal of Central South University (Science and Technology), 2011,42(9):2853-2859. 